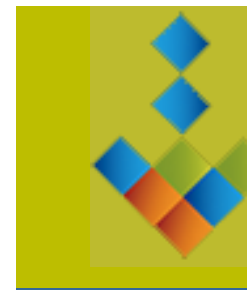

RITPU|IJTHE

ritpu.org | ijthe.org

Revue internationale des technologies en pédagogie universitaire International Journal of Technologies in Higher Education

\title{
Le numérique, plus que jamais au service de la mission d'enseignement des cégeps
}

Bernard TREMBLAY bernard.tremblay@fedecegeps.qc.ca Président-directeur général Fédération des cégeps

\section{How Education Technology Became Central to the Teaching Mission of Colleges (CEGEPs) in Quebec}

https://doi.org/10.18162/ritpu-2020-v17n1-05

En mars dernier, lorsque le gouvernement a annoncé la reprise des cours après deux semaines de suspension des activités des cégeps, l'ensemble du personnel des 48 établissements du réseau collégial public du Québec est d'un seul coup passé à une vitesse supérieure en matière d'intégration du numérique. Si nul ne peut affirmer être totalement prêt à réagir en temps de pandémie, les cégeps ont déployé leur agilité habituelle pour prendre à très grande vitesse ce virage numérique et poursuivre leur mission d'enseignement.

Bien sûr, le numérique faisait déjà partie de l'environnement collégial. Les répondantes et répondants TIC, regroupés au sein du Réseau REPTIC ${ }^{1}$, agissaient et militaient depuis longtemps en faveur d'une plus grande utilisation des technologies dans l'enseignement. Plusieurs organismes du réseau collégial - Profweb, APOP, FADIO, Performa, DECclic, la Vitrine technologie-éducation, Cégep à distance, etc. - travaillaient depuis plusieurs années au développement de la technopédagogie et de l'enseignement à distance. À l'échelle provinciale, des travaux visant la mise en œuvre d'un eCampus étaient en cours et les cégeps s'étaient préalablement montrés proactifs en proposant au gouvernement des pistes prometteuses lors de l'élaboration de la Stratégie numérique du gouvernement du Québec, dont découle le Plan d'action numérique (PAN) avec ses 33 mesures. L'enjeu du numérique figurait donc parmi les priorités des cégeps, qui ont réclamé, par la voix de la Fédération des cégeps, des ressources supplémentaires pour accélérer l'implantation des technologies dans leurs établissements. Mais, quoi qu'il en soit, jusqu'en mars dernier, dans le réseau collégial, l'utilisation des technologies dans l'enseignement variait en intensité d'un milieu à l'autre, voire d'un programme ou département à l'autre.

1. Le Réseau REPTIC (http://reptic.qc.ca) est une communauté de pratique qui regroupe, anime et appuie les conseillères et conseillers pédagogiques responsables de l'intégration pédagogique des technologies dans les cégeps et les collèges publics et privés du Québec. Il est rattaché à la Fédération des cégeps. 
La pandémie et l'obligation qu'elle amenait d'offrir les cours à distance uniquement allaient venir créer une véritable explosion de l'activité numérique dans les cégeps. À titre d'exemple, alors qu'environ 3000 enseignantes et enseignants utilisaient l'application Moodle, ce nombre était passé à 4500 quelques semaines après la reprise des cours! Les technologies, qui pouvaient parfois être perçues comme accessoires dans une stratégie d'enseignement, venaient d'atteindre le statut d'outil essentiel. En fait, dans le contexte, le numérique a carrément sauvé la mission d'enseignement des cégeps, et cela, même si tout n'était pas parfait et que tous conviennent que les technologies ne répondent pas à l'ensemble des besoins des étudiants et étudiantes et ne remplacent pas entièrement la présence, ni en classe ni dans ce milieu de vie enrichissant que sont les établissements. En clair, le numérique est absolument essentiel au collégial, mais il n'est pas une panacée.

Rapidement, le personnel enseignant a dû revoir ses pratiques et ses stratégies d'enseignement pour prendre appui sur une multitude d'outils, avec l'aide du personnel professionnel qui a notamment soutenu l'utilisation des plateformes technologiques existantes soudainement mises à rude épreuve en raison de l'augmentation massive de la demande. Le serveur Web, le serveur de base de données et plusieurs autres composantes devaient être paramétrés et coordonnés, ce qui a demandé des efforts gigantesques au cours des premières semaines. Heureusement, l'infrastructure technologique était bien implantée, et le réseau de communications bien organisé a permis à chaque répondante ou répondant TIC de se mettre au service des enseignants et enseignantes de tous les collèges. La réponse au problème d'un établissement pouvait aussi s'appliquer dans un autre, un avantage du fonctionnement en réseau des 48 cégeps.

Les autres catégories de personnel ainsi que les gestionnaires des collèges ont tous redoublé d'efforts dans leur champ d'expertise respectif pour faire en sorte que les étudiantes et étudiants bénéficient des meilleures conditions possible d'apprentissage, dans un contexte unique et exigeant. C'est donc un effort collectif qui aura permis de préserver la session d'hiver au cégep, de concrétiser le virage numérique dans le réseau collégial, voire de convertir au passage les moins convaincus et, surtout, de mieux outiller les cégeps pour la rentrée de l'automne prochain et les années à venir.

Dans cet effort collectif, il ne faut surtout pas oublier celui des étudiants et étudiantes. Car, aussi important que soit l'enjeu du numérique, dans le réseau collégial comme dans les autres sphères de notre société, l'utilisation accélérée des technologies en enseignement a fait ressortir l'enjeu humain. Le fait qu'ils maîtrisent l'utilisation des médias sociaux dans un contexte de loisir, par exemple, ne veut pas dire que les jeunes soient nécessairement prêts à suivre des cours à distance à l'enseignement supérieur. De nouvelles compétences ont dû être intégrées, des aptitudes ont été développées, bref la population étudiante des cégeps s'est aussi vu imposer de nouveaux défis en matière d'apprentissage comme d'utilisation des outils technologiques. Devant ces nouveaux modes d'apprentissage, et avec les effets psychologiques de l'isolement, certains ont pu se détourner de leurs études ou éprouver plus de difficulté qu'à l'habitude. La persévérance scolaire est par conséquent un important sujet de préoccupation, en éducation en général et au collégial en particulier.

Le fait de miser presque uniquement sur les technologies a aussi fait ressortir des inégalités socioéconomiques. Certains étudiants et étudiantes ne disposent pas comme d'autres de leur propre ordinateur et le Québec compte encore des régions qui ne bénéficient pas d'une bonne couverture Internet. 
Le numérique en tant que véhicule d'apprentissage est bien implanté et il occupera assurément une place grandissante dans l'avenir. Mais, au-delà de l'implantation des infrastructures technologiques nécessaires, il faudra s'assurer que tous puissent continuer d'avoir des chances égales d'accéder à l'enseignement collégial, puisqu'il s'agit là d'une motivation ayant présidé à la création du réseau des cégeps, qui a fait faire des bonds de géant au Québec en matière de scolarisation. 